\title{
Eventos vitales estresantes, estrategias de afrontamiento y resiliencia en adolescentes en contexto de pandemia
}

\section{Stressful life events, coping strategies and adolescent resilience in the context of a pandemic}

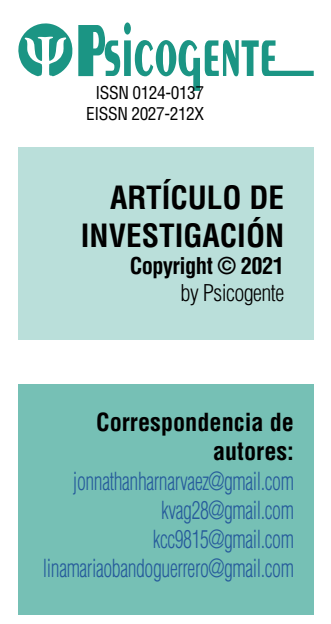

Recibido: $17-12-20$ Aceptado: 02-09-21 Publicado: $01-10-21$

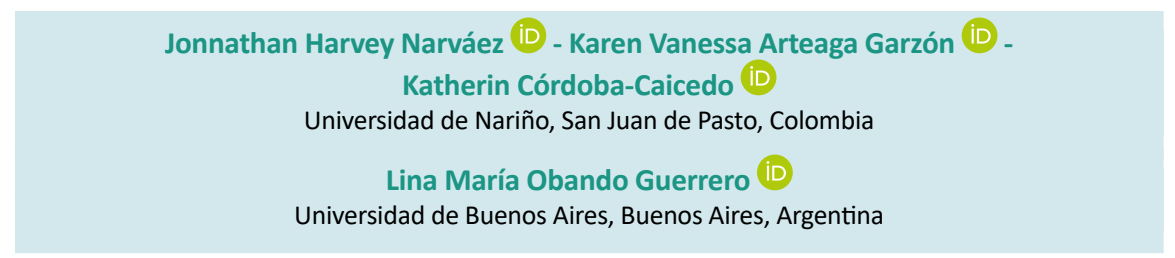

Resumen

Introducción: El impacto psicológico causado por la pandemia Covid-19 en los adolescentes escolarizados conlleva la necesidad del análisis de las estrategias de afrontamiento y la resiliencia, al momento de enfrentar los eventos vitales estresantes.

Objetivo: Identificar la correlación entre las variables eventos vitales estresantes, estrategias de afrontamiento y resiliencia en un grupo de adolescentes de la ciudad de Pasto, Colombia en el contexto de la Pandemia Covid-19.

Método: Estudio descriptivo correlacional, no experimental transversal. Se aplicaron las versiones adaptadas del Cuestionario de Eventos Vitales Estresantes CEVE-A-R para Adolescentes (Villalobos et al., 2011), la Escala Estrategias de Coping - Modificada - EEC-M (Londoño et al., 2006) y la Escala de Resiliencia Escolar - E.R.E (Obando et al., 2020). Se seleccionó una muestra intencional de 145 adolescentes entre hombres y mujeres con edades comprendidas entre los 14 y 19 años.

Resultados: La variable eventos vitales estresantes se correlacionó de forma inversa estadísticamente significativa con resiliencia $(p=-0,250)$ y de manera directa con las estrategias de afrontamiento tales como evitación emocional $(p=0,164)$, reacción agresiva $(p=0,228)$ y expresión de la dificultad de afrontamiento $(p=0,254)$. Por el contrario, se encontró una correlación inversa con las estrategias de solución de problemas $(p=-0,172)$ y búsqueda de apoyo social $(p=-0,04)$.

Conclusiones: Se identifica en población adolescente en tiempos de pandemia una correlación débil entre las variables eventos vitales estresantes y estrategias de afrontamiento, y una correlación moderada entre resiliencia y las estrategias de solución de problemas y búsqueda de apoyo social.

Palabras clave: estrategias de afrontamiento; estrés vital; resiliencia psicológica; adolescente (DeCS)

Abstract

Introduction: The psychological impact caused by the Covid-19 pandemic on adolescents in school entails the need for analysis of coping strategies and resilience, when facing stressful life events.

Objective: To identify the correlation between the variables of stressful life events, coping strategies and resilience in a group of adolescents from the city of Pasto, Colombia in the context of the Covid-19 Pandemic.

Method: descriptive, correlational, non-experimental cross-sectional study. The adapted versions of the CEVE-AR Stressful Life Events Questionnaire for Adolescents (Villalobos et al., 2011), the Coping Strategies Scale- Modified- EEC-M (Londoño et al., 2006) and the School Resilience Scale were applied - E.R.E (Obando et al., 2020). An intentional sample of 145 adolescents between men and women aged between 14 and 19 years was selected.

Results: The stressful life events variable was statistically significantly inversely correlated with resilience $(p=-0,250)$ and directly with coping strategies such as emotional avoidance $(p=0,164)$, aggressive reaction $(p=0,228)$ and expression of coping difficulty $(p=0,254)$. On the contrary, an inverse correlation was found with problem solving strategies $(p=-0,172)$ and seeking social support $(p=-0,04)$.

Cómo citar este artículo (APA):

Harvey Narváez, J., Arteaga Garzón, K. V., Córdoba-Caicedo, K. \& Obando Guerrero, L. M. (2021). Eventos vitales estresantes, estrategias de afrontamiento y resiliencia en adolescentes en contexto de pandemia. Psicogente 24(46), 1-17. https://doi.org/10.17081/psico.24.46.4789 
Conclusions: A weak correlation between stressful life events and coping strategies, and a moderate correlation between resilience and problem-solving strategies and seeking social support, is identified in the adolescent population in times of pandemic.

Keywords: coping strategies; life stress; resilience psychological; adolescent (DeCS)

\section{INTRODUCCIÓN}

La Organización Mundial de la Salud (2020) declaró oficialmente el inicio de una pandemia a causa del virus SARS-CoV-2 que trajo consecuencias mundiales en la economía, la salud, la educación y los contextos sociales. En relación a la salud mental o psicológica Sandín, García \& Chorot (2020) y Shigemura, Ursano, Morganstein \& Benedek (2020) reconocieron que, a causa de la incertidumbre, la reducción de la autonomía y las preocupaciones constantes que surgieron a raíz de la pandemia, se desarrollaron respuestas de estrés, ansiedad, depresión, alteraciones emocionales y del sueño que repercuten en el bienestar de la población.

Asimismo, en el sector educativo tras la suspensión de clases presenciales se aceleró el uso de la tecnología como metodología de enseñanza - aprendizaje, transformando los procesos educativos tradicionales que produjo repercusiones negativas para aquellos que no estaban familiarizados con los medios virtuales (Montes, 2020). Junto a ello, se identifica un drástico cambio de rutinas y hábitos tanto para los estudiantes como para los padres, quienes al compartir el mismo espacio geográfico, se pueden ver en la obligación de participar en los escenarios escolares, eso sumado al trabajo en casa que afecta las relaciones y convivencia familiar (Lorenzo, Díaz \& Zaldívar, 2020).

A nivel social, el distanciamiento que se implementó para disminuir la propagación del virus ha afectado las relaciones interpersonales entre los sujetos. En el caso de los adolescentes se vieron expuestos a la pérdida de relaciones sociales con pares, actividades extensas de ocio, dinámicas de vida sedentarias, limitación del espacio de interacción, entre otros (Espada, Orgilés, Piqueras \& Morales, 2020). Estas modificaciones en la vida cotidiana junto con los cambios físicos y psicológicos que experimentan los adolescentes generan alteraciones emocionales y conductuales (Tremolada, Bonichini \& Taverna, 2016; Reyes, Reséndiz, Alcázar \& Reidl, 2017; Uribe, Ramos, Villamil \& Palacio, 2018).

De esta manera, se ha identificado que las consecuencias de la pandemia han desencadenado una serie de factores de riesgo del estrés psicosocial, en el cual las personas que perciben esta situación de alta relevancia 
como incontrolable pueden verse particularmente afectadas (Leiva, Nazar, Martínez, Petermann, Ricchezza \& Celis, 2020). Por consiguiente, se puede evidenciar cómo los fenómenos relacionados con la pandemia han expuesto de manera repentina a los adolescentes ante diversos eventos vitales estresantes, entendidos como situaciones incontrolables, que surgen de manera impredecible, debido a cambios ambientales o sociales que se presentan de manera observable o discreta y que representan un alto grado de relevancia en la vida de las personas, alterando el equilibrio interno de cada individuo. Estos eventos pueden ser normativos, los cuales implican la transición de una etapa a otra, por ejemplo, la adolescencia; o no normativos referentes a situaciones externas como la separación de los padres, enfermedades o fallecimientos (García y Acuña, 2016; Rey, 2015).

Por otra parte, el afrontamiento del estrés psicosocial produce la necesidad de un reajuste de las diferentes condiciones a las que se ven expuestos los adolescentes en especial a las que ha dejado la pandemia del covid-19 (Delgado y Santacruz, 2011; Pérez, Alcázar \& Reidl, 2020). Es así, como el afrontamiento, involucra respuestas cognitivas, emocionales y conductuales que el individuo deberá emplear para contrarrestar una situación percibida como estresante en un escenario particular de la vida cotidiana que requiere un esfuerzo de adaptación que cada individuo asume en función de los estilos de afrontamiento (Castaño y del Barco, 2010; Viñas, Malo, González, \& Navarro, 2015).

De acuerdo a Solis y Vidal (2006) cada estilo de afrontamiento está constituido por unas estrategias de afrontamiento. De esta manera los autores hacen un recorrido por las diferentes clasificaciones y las agrupan en tres estilos a) en el estilo productivo se encuentran las estrategias búsqueda de apoyo social, concentrarse en resolver el problema, recreación física, diversiones relajantes, invertir en amigos cercanos, buscar pertenecer, trabajar duro y tener éxito, fijarse en lo positivo; b) en el estilo no productivo se destacan las estrategias de evitación como preocuparse, buscar pertenecer, hacerse ilusiones, no hacer frente, ignorar el problema, reducción de la tensión, reservarlo para sí mismo, autoinculparse; c) por último en el estilo de afrontamiento en referencia a otros, se encuentra la búsqueda de apoyo social, espiritual, de ayuda profesional y acción social.

Teniendo en cuenta que los adolescentes en tiempos de pandemia se han visto expuestos a todo tipo de cambios en cuanto a sus ámbitos educativo, familiar, comunitario, sentimental, y otros (Montes, 2020) es necesario reconocer qué estrategias de afrontamiento emplearon para sobrellevar 
esta situación de crisis y cuál fue el impacto en su adaptación a la nueva normalidad, ya que esto le permite a los lectores en especial a profesionales de la psicología y pedagogía, identificar y fortalecer a mediano y largo plazo las estrategias de afrontamiento que han mostrado mayor efectividad en la mitigación de estresores psicosociales. En esta lógica, es importante que los adolescentes activen factores protectores internos y externos al momento de restablecer los procesos que les permiten sobreponerse a las nuevas demandas académicas, sociales y familiares, así como a los factores de riesgo externos e internos que podrían generar dificultades en el aprendizaje (Coronado, 2016). También se analiza la variable resiliencia entre tantos estudios como los de Gómez y Rivas (2017) dan cuenta que las personas con mayores niveles de resiliencia, tienen mayor capacidad para adaptarse a situaciones adversas, y ante situaciones traumáticas o estresores sostenidos, y presentan altos niveles de estabilidad y mayor capacidad de adaptación.

En efecto, el presente artículo tiene como objetivo identificar la correlación entre las variables eventos vitales estresantes, estrategias de afrontamiento y resiliencia que presentan un grupo de adolescentes de una institución educativa de la ciudad de Pasto quienes se han visto afectados por el impacto psicosocial de la pandemia Covid-19.

\section{MÉTODO}

\subsection{Diseño}

El presente estudio es descriptivo correlacional, no experimental transversal. Es no experimental dado que no hay una manipulación intencional de las variables, por el contrario, se buscó medir y caracterizar los eventos vitales estresantes, las estrategias de afrontamiento y la resiliencia de un grupo de adolescentes. Una vez descritos los constructos se evaluó si existía correlación entre ellos en un momento determinado por ende fue un estudio transversal (Álvarez, 2020; Hernández, Fernández \& Baptista, 2014).

\subsection{Participantes}

Dada la contingencia actual, para el desarrollo del presente estudio se trabajó con una muestra por conveniencia constituida por 145 estudiantes que cumplieron con los criterios de inclusión establecidos por los investigadores. Entre ellos se destacaron: a) ser adolescentes entre los 11 a 19 años, b) tener matrícula regular vigente en la Institución Educativa Ciudad de Pasto y c) pertenecer a los estratos socioeconómicos 1,2 y 3, d) contar con 
acceso a Internet. Como criterio de exclusión, no se trabajó con estudiantes que no tenían el asentimiento y consentimiento informado debidamente diligenciados.

\subsection{Instrumentos para la recolección de información}

\subsubsection{Cuestionario de Eventos Vitales Estresantes CEVE-A-R para}

Adolescentes (Villalobos, Delgado, Santacruz \& Timarán, 2011).

La versión adaptada en Colombia del CEVE-A-R quedó constituida por 26 ítems independientes, que buscaron evaluar los eventos vitales estresantes que vivieron los adolescentes en los últimos seis meses. La primera parte del instrumento fue dicotómica en la cual los sujetos respondieron sí (1) o no (0), dependiendo si vivió o no el evento vital. Por otra parte, el sujeto determinó el grado de afectación que el evento tuvo en su vida mediante una escala Likert en un rango de frecuencia de 0 a 2 desde Nada a Mucho. Frente al instrumento se encuentran dos medidas diferentes de calificación; por un lado el promedio total de eventos estresantes vividos por los adolescentes y por otro, el promedio del grado de afectación que estos eventos estresantes vitales tuvieron en ellos.

\subsubsection{Escala Estrategias de Coping - Modificada (EEC-M) (Londoño,} Henao, Puerta, Posada, Arango, Aguirre \& Acevedo 2006).

La escala brindó información sobre el conjunto de recursos y esfuerzos cognitivos y comportamentales que las personas emplearon, para orientarlos a resolver un problema, a reducir o eliminar la respuesta emocional o a modificar la evaluación inicial de la situación (Londoño et al., 2006).

La versión adaptada en Colombia de la EEC-M contiene 69 ítems, con opciones de respuesta tipo Likert, que van en un rango de frecuencia de 1 a 6 desde Nunca hasta Siempre. Cuenta con 12 dimensiones distribuidas de la siguiente manera: 9 ítems para la dimensión solución de problemas, 7 ítems para apoyo social, 9 ítems para espera, 7 ítems para religión, 8 ítems para evitación emocional, 5 ítems para búsqueda de apoyo profesional, 5 ítems para reacción agresiva, 5 ítems para evitación cognitiva, 4 ítems para expresión de la dificultad de afrontamiento, 5 ítems para reevaluación positiva, 3 items para negación y 2 ítems para autonomía. Cabe mencionar que no existen ítems invertidos en la versión adaptada en Colombia por lo que la puntuación es directa, además se hace hincapié en que cada dimensión tiene una cantidad de ítems diferente por lo que para su calificación y comparación 
se sacó el promedio de cada subcategoría y se lo dividió entre el número de ítems. El Alfa de Cronbach fue de 0,85 y la varianza total explicada del $58 \%$ (Londoño et al., 2006).

\subsubsection{Escala de Resiliencia Escolar- E.R.E. (Obando, Narváez \& Crespi,} 2020).

La escala fue construida en Chile, teniendo como base los fundamentos teóricos y prácticos de la Escala SV- RES construida por Saavedra y Villalta (2008). La versión adaptada para Colombia está constituida por 26 ítems, con cinco opciones de respuesta que van de muy de acuerdo (5) a muy en desacuerdo (1). Está compuesta por dos dimensiones que hacen alusión a los recursos internos (ítems directos 1,2,3,7,8,15,16,25,26) que se relacionan con la autoestima, el optimismo respecto al futuro y la consecución de metas y los recursos externos (ítems directos 4,5,6,9,10,11,12,13,14,17,18,19,20, $21,22,23,24)$ vinculados con la satisfacción con la vida, el apoyo social, las redes-modelos y la comunicación y expresión de sentimientos. Teniendo en cuenta que, la cantidad de ítems es diferente para cada dimensión, para su calificación se sacó el promedio de cada subcategoría y se lo dividió entre el número de ítems. Respecto a la escala general para calificarla, se debe hacer una sumatoria total de ítems. El alfa ordinal de la escala fue de 0,92.

\subsection{Procedimiento}

Inicialmente, se presentó el proyecto de investigación a las directivas de la Institución Educativa con el fin de obtener la respectiva autorización para la ejecución del estudio, el cual se realizó del mes de mayo al mes de julio del 2020. Con la autorización otorgada y teniendo en cuenta que, por la contingencia actual no se podían aplicar los instrumentos de forma presencial, se diseñó un protocolo para su administración de forma virtual. De este modo, se citó a reunión por Zoom a padres y madres de familia para explicar el objetivo de la investigación y la importancia de que los hijos y/o hijas puedan participar de la misma. En este espacio se aclararon dudas, se explicaron los posibles riesgos, se solicitó la firma del consentimiento y asentimiento informado y se hizo hincapié de que para la administración del instrumento se requería del uso de Internet. A las personas que decidieron participar del proceso, se les envió un audio expositivo en el que se explicaba la forma de realizar el instrumento, su duración y que junto con el correo se enviaba el enlace con los instrumentos los cuales tenían preguntas que se debían responder en función al contexto de la pandemia. Por su parte, las personas 
que decidieron no participar del estudio argumentaban que se debía por la dificultad al acceso a Internet, el poco tiempo de los padres debido a sus trabajos lo que les impedía acompañar esta actividad y la falta de interés. Con los resultados obtenidos se procedió a realizar los análisis estadísticos y se sintetizó toda la información en un informe final, garantizando la devolución de emergentes al establecimiento educativo mediante un informe técnico.

\subsection{Análisis de datos}

Inicialmente se realizó un análisis descriptivo para cada una de las variables objeto de estudio, de modo que se evaluó la media, desviación estándar, puntaje mínimo y máximo. Posteriormente, se ejecutó la prueba de normalidad Kolmogorov-Smirnov por variables estudiadas para determinar la distribución de los datos. Teniendo en cuenta que los datos fueron no paramétricos se aplicó el coeficiente Rho de Spearman. Se plantea que un valor $r$ cercano a 1 indica que la relación es positiva y fuerte, por el contrario, si el valor se aproxima a -1 representa una relación negativa y fuerte. Asimismo, si la relación está próxima a 0,5 se considera que es una relación moderada y positiva y si está cercano a -0,5 se trata de una relación moderada y negativa. Si el valor se aproxima a 0 refleja una correlación débil (Burbano y Guerrero, 2021). En esta lógica se correlacionaron las variables eventos vitales estresantes, resiliencia académica, y estrategias de afrontamiento. El análisis de los datos se realizó mediante el software IBM SPSS Stactistics Standard versión 25,0 .

Cabe mencionar que para el desarrollo de la investigación se siguieron los parámetros éticos establecidos en la Ley 1090 de 2006 (Congreso de Colombia, 2006), en la cual se instituye el Código Deontológico y Bioético del profesional de la psicología en Colombia, que establece que el manejo de información es estrictamente confidencial. Se contó además con la autorización de los padres de familia, quienes diligenciaron y firmaron el consentimiento informado, y de los estudiantes, quienes una vez autorizados, asintieron su participación en el estudio.

\section{RESULTADOS}

El presente estudio quedó constituido por una muestra de 145 estudiantes. La Tabla 1 contempla las características sociodemográficas en donde se destaca la prevalencia del sexo femenino $(55,9 \% \mathrm{n}=81)$, con edades comprendidas entre los 16 a 17 años $(73,1 \% n=106)$, que pertenecen al estrato socioeconómico bajo-bajo ( $49 \% \mathrm{n}=71$ ) con prevalencia de tipo de familia nuclear $(44,8 \% n=65)$. 
Tabla 1.

Características sociodemográficas de la muestra

\begin{tabular}{ccc}
\hline & \multicolumn{1}{c}{ SEXO } \\
\hline Masculino & \\
Femenino & $44,1 \%(64)$ \\
& EDAD & $55,9 \%(81)$ \\
\hline 14 a 15 años & $17,2 \%(25)$ \\
16 a 17 años & $73,1 \%(106)$ \\
18 a 19 años & $9,7 \%(14)$ \\
\hline \multicolumn{1}{c}{ ESTRATO SOCIOECONÓMICO } \\
\hline Bajo-Bajo & $49 \%(71)$ \\
Bajo & $37,9 \%(55)$ \\
Medio-Bajo & $13,1 \%(19)$ \\
\hline \multicolumn{2}{c}{ TIPO DE FAMILIA } \\
\hline Monoparental & $26,9 \%(39)$ \\
Nuclear & $44,8 \%(65)$ \\
Extensa & $26,9 \%(39)$ \\
Reconstituida & $1,4 \%(2)$ \\
\hline
\end{tabular}

En cuanto al análisis descriptivo de las variables eventos vitales estresantes, resiliencia académica y estrategias de afrontamiento, los estudiantes obtuvieron una media de 6,79 y desviación estándar de 3,89 en eventos vitales estresantes, el puntaje mínimo fue de 0 y el máximo de 20 . En lo que respecta a la resiliencia los sujetos obtuvieron una media de 72,80 con desviación estándar de 15, el puntaje mínimo fue de 13 y el máximo de 104. Frente a las estrategias de afrontamiento se encontró que las estrategias más empleadas por los adolescentes fueron evitación emocional $(2,82 \%)$, solución de problemas (2,4\%), evitación cognitiva (2,34 \%) y expresión de la dificultad de afrontamiento (2,32 \%). En menor medida los estudiantes emplean estrategias de afrontamiento como la búsqueda de apoyo profesional $(0,82 \%)$ y la búsqueda de apoyo social (1,69\%).

\subsection{Eventos vitales estresantes y estrategias de afrontamiento}

Por otra parte, en el estudio se buscó determinar la relación entre eventos vitales estresantes y resiliencia, y la relación de estos constructos con las estrategias de afrontamiento. Para ello se aplicó la prueba de normalidad Kolmogorov-Smirnov encontrando un valor $p$ de 0,001 lo que significa que los datos no se distribuyen de forma normal por lo que se aplicaron pruebas no paramétricas. De este modo, se utilizó el coeficiente de correlación de Spearman. 
En la Tabla 2 se expone que, si bien la correlación es baja, los eventos vitales estresantes y las estrategias de afrontamiento tales como evitación emocional, reacción agresiva y expresión de la dificultad de afrontamiento, tienen una correlación directa estadísticamente significativa. En este sentido se puede plantear que, frente a los eventos vitales estresantes percibidos en tiempo de pandemia, los estudiantes prefieren evitar expresar sus emociones y posiblemente tengan dificultades para afrontar las situaciones lo que puede derivarse en conductas agresivas. Por el contrario, se encontró una correlación inversa con la solución de problemas y búsqueda de apoyo social, que si bien es estadísticamente significativa su fuerza de correlación es muy baja, por lo que ante un evento vital estresante ocasionado por la pandemia, los estudiantes pueden o no buscar apoyo para solucionar los problemas.

Tabla 2.

Correlación entre estrategias de afrontamiento y eventos vitales estresantes

\begin{tabular}{|c|c|c|c|c|c|c|c|c|c|c|c|c|c|}
\hline & EVE & $S P$ & BAS & ESP & REL & EE & BAP & RA & EC & RP & EDA & $\mathrm{N}$ & A \\
\hline EVE & 1,00 &,$- 172^{*}$ & $-0,04^{*}$ & 0,08 & 0,09 & ,164* & 0,00 &, $228 * *$ & $-0,15$ & $-0,09$ &, $254^{* *}$ & 0,01 & $-0,07$ \\
\hline$S P$ & & 1,00 &, $413^{* *}$ & $-0,08$ & ,206* & $-0,14$ &, $332^{* *}$ & $-0,08$ &, $251^{* *}$ &, $563^{* *}$ &,$- 232^{* *}$ & 0,00 & $-0,02$ \\
\hline BAS & & & 1,00 & $-0,13$ &, $347^{* *}$ &,$- 394^{* *}$ &, $568^{* *}$ & 0,14 &, $232 * *$ &, $313 * *$ &,$- 248^{* *}$ & 0,02 &,$- 266^{* *}$ \\
\hline ESP & & & & 1,00 & 0,02 & ,200* & $-0,05$ & 0,16 & 0,13 & 0,08 &, $225^{* *}$ &, $244^{* *}$ & $176^{*}$ \\
\hline REL & & & & & 1,00 & 0,00 &, $219 * *$ &, $275^{* *}$ & 0,08 &, $223 * *$ & $-0,06$ &,$- 187^{*}$ &,$- 169 *$ \\
\hline $\mathrm{EE}$ & & & & & & 1,00 &,$- 342^{* *}$ & 0,05 & 0,03 & $-0,10$ &, $636^{* *}$ & 0,03 &, $334^{* *}$ \\
\hline BAP & & & & & & & 1,00 & 0,16 & 0,10 &, $219 * *$ &,$- 171^{*}$ & $-0,08$ &,$- 242 * *$ \\
\hline RA & & & & & & & & 1,00 & $-0,04$ & $-0,08$ & ,203* & 0,03 & 0,09 \\
\hline$E C$ & & & & & & & & & 1,00 &, $397 * *$ & $-0,06$ &, $467 * *$ & 0,16 \\
\hline $\mathrm{RP}$ & & & & & & & & & & 1,00 & $-0,14$ & ,184* & 0,06 \\
\hline EDA & & & & & & & & & & & 1,00 & 0,01 & ,177* \\
\hline $\mathrm{N}$ & & & & & & & & & & & & 1,00 &, $354^{* *}$ \\
\hline A & & & & & & & & & & & & & 1,00 \\
\hline
\end{tabular}

Nota: $\mathrm{N}=145 ; * * \mathrm{p}<0,01 ; * \mathrm{p}<0,05 . \mathrm{EVE}=$ Eventos Vitales Estresantes, $\mathrm{SP}=$ Solución de Problemas, BAS= Búsqueda de Apoyo Social, ESP=Espera, REL=Religión, EE= Evitación Emocional, $\mathrm{BAP}=\mathrm{Bú}$ squeda de Apoyo Profesional, $\mathrm{RA}=$ Reacción Agresiva, EC= Evitación Cognitiva, $\mathrm{RP}=$ Reevaluación Positiva, EDA=Expresión de la Dificultad de Afrontamiento, $\mathrm{N}=$ Negación, A=Autonomía

\subsection{Eventos vitales estresantes y resiliencia}

Frente a la correlación entre eventos vitales estresantes y resiliencia se encontró una relación inversa estadísticamente significativa entre las variables la cual es baja (ver Tabla 3). En este sentido, se identificó que un segmento poblacional de los adolescentes presentaba niveles inferiores en los puntajes de resiliencia y mayores niveles de eventos vitales estresantes en la pandemia, de modo que, posiblemente la resiliencia puede ser una de las variables protectoras para que los adolescentes se sobrepongan a la contingencia actual. 
Tabla 3.

Correlación entre las variables eventos vitales estresantes y resiliencia

\begin{tabular}{cc}
\hline & EVENTOS VITALES ESTRESANTES \\
Resiliencia & $-0,250^{* *}$ \\
\hline \multicolumn{2}{c}{ Nota: $\mathrm{N}=145 ;{ }^{* *} \mathrm{p}<0,01 ;{ }^{*} \mathrm{p}<0,05$}
\end{tabular}

\subsection{Estrategias de afrontamiento y resiliencia}

En cuanto a la correlación entre las estrategias de afrontamiento y resiliencia (Tabla 4) se encontró que existe una correlación directa estadísticamente significativa y moderada con solución de problemas, búsqueda de apoyo social, religión, búsqueda de apoyo profesional, evitación cognitiva y reevaluación positiva. En consecuencia, los adolescentes con mayores niveles de resiliencia tienden en la pandemia a analizar las causas del problema y generar soluciones, comunican el malestar generado por el estresor y buscan apoyo profesional o social, neutralizan los efectos de los pensamientos negativos mediante la distracción o la negación o identifican los efectos positivos de la pandemia y aprenden de las circunstancias. En contraposición, se identificaron correlaciones inversas estadísticamente significativas bajas con las dimensiones espera y autonomía y asociaciones moderadas con evitación emocional y expresión de la dificultad de afrontamiento. En este sentido, los estudiantes con estrategias de evitación emocional otorgan gran carga emocional al estresor y evitan expresar sus emociones, además de excluir factores externos tan importantes como las redes de apoyo social que proveen recursos sustanciales al momento de afrontar de manera efectiva cualquier situación de crisis.

Tabla 4.

Correlación entre estrategias de afrontamiento y resiliencia

\begin{tabular}{|c|c|c|c|c|c|c|c|c|c|c|c|c|c|}
\hline & RES & SP & BAS & ESP & REL & $\mathrm{EE}$ & BAP & RA & EC & RP & EDA & $\mathrm{N}$ & A \\
\hline RES & 1,00 &, $379 * *$ &, $587^{* *}$ &,$- 218^{* *}$ &, $266 * *$ &,$- 485^{* *}$ &, $381^{* *}$ & $-0,12$ &, $263^{* *}$ &, $380 * *$ &,$- 407^{* *}$ & $-0,05$ &,$- 292 * *$ \\
\hline$S P$ & & 1,00 &, $413^{* *}$ & $-0,08$ & ,206* & $-0,14$ &, $332 * *$ & $-0,08$ &, $251^{* *}$ &, $563^{* *}$ &,$- 232 * *$ & 0,00 & $-0,02$ \\
\hline BAS & & & 1,00 & $-0,13$ &, $347^{* *}$ &,$- 394^{* *}$ &, $568 * *$ & 0,14 &, $232 * *$ &, $313^{* *}$ &,$- 248^{* *}$ & 0,02 &,$- 266 * *$ \\
\hline ESP & & & & 1,00 & 0,02 & ,200* & $-0,05$ & 0,16 & 0,13 & 0,08 &, $225^{* *}$ &, $244 * *$ &, $176^{*}$ \\
\hline REL & & & & & 1,00 & 0,00 &, $219 * *$ &, $275^{* *}$ & 0,08 &, $223 * *$ & $-0,06$ &,$- 187^{*}$ &,$- 169 *$ \\
\hline $\mathrm{EE}$ & & & & & & 1,00 &,$- 342 * *$ & 0,05 & 0,03 & $-0,10$ &, $636 * *$ & 0,03 &, $334^{* *}$ \\
\hline BAP & & & & & & & 1,00 & 0,16 & 0,10 &, $219 * *$ &,$- 171^{*}$ & $-0,08$ &,$- 242 * *$ \\
\hline RA & & & & & & & & 1,00 & $-0,04$ & $-0,08$ & ,203* & 0,03 & 0,09 \\
\hline $\mathrm{EC}$ & & & & & & & & & 1,00 &, $397 * *$ & $-0,06$ &, $467^{* *}$ & 0,16 \\
\hline $\mathrm{RP}$ & & & & & & & & & & 1,00 & $-0,14$ & ,184* & 0,06 \\
\hline EDA & & & & & & & & & & & 1,00 & 0,01 &, $177^{*}$ \\
\hline $\mathrm{N}$ & & & & & & & & & & & & 1,00 &, $354^{* *}$ \\
\hline A & & & & & & & & & & & & & 1,00 \\
\hline
\end{tabular}

Nota: $\mathrm{N}=145 ; * * \mathrm{p}<0,01 ; * \mathrm{p}<0,05$. RES= Resiliencia, $\mathrm{SP}=$ Solución de Problemas, BAS= Búsqueda de Apoyo Social, $\mathrm{ESP}=$ Espera, $\mathrm{REL}=$ Religión, $\mathrm{EE}=$ Evitación Emocional, $\mathrm{BAP}=$ Búsqueda de Apoyo Profesional, $\mathrm{RA}=$ Reacción Agresiva, $\mathrm{EC}=$ Evitación Cognitiva, $\mathrm{RP}=$ Reevaluación Positiva, EDA=Expresión de la Dificultad de Afrontamiento, $\mathrm{N}=\mathrm{Negación}$ A=Autonomía 


\section{DISCUSIONES}

A lo largo del proceso investigativo se reconoce que los adolescentes tuvieron que atravesar múltiples cambios en su vida, tales como la pérdida de relaciones sociales, cambios en sus actividades de ocio, transformación de las rutinas académicas, entre otros eventos vitales que impactaron las relaciones sociales, personales, escolares y familiares; lo que también de acuerdo a algunos estudios contribuyó al fortalecimiento de capacidades psicológicas y recursos familiares, situación que favoreció en algunos casos la mitigación del impacto psicosocial negativo de la pandemia (Espada et al., 2020). En este aspecto, el estudio da cuenta del despliegue de estilos de afrontamiento que favorecen el control de los estresores emergentes y la adaptación a los efectos del confinamiento, lo que corrobora los hallazgos de Prime, Wade, \& Browne (2020) quienes reconocen que los adolescentes en el contexto de Covid-19, desplegaron una serie de procesos adaptativos, entre ellos, el fortalecimiento de relaciones positivas dentro de la familia y la habilitación de fuentes de soporte y apoyo familiar.

En cuanto a la percepción de estrés el estudio indica una afectación media-baja en los eventos vitales estresantes, sin embargo, la evidencia de abordajes muestrales a gran escala dan cuenta que la población adolescente experimentó de manera sistemática y sucesiva situaciones de estrés en medio de la cuarentena, entre los más relevantes, el aislamiento en el hogar, las clases virtuales y la disminución de las interacciones sociales, eventos que incidieron en el proceso de adaptación, desarrollo y funcionamiento social siendo evidentes los impactos negativos en la salud (Alianza para la Protección de la Infancia y adolescencia en la Acción Humanitaria, 2020), lo que contrasta con el informe científico del Ministerio de salud y Protección social (2020) el cual consideró que los adolescentes tienen mayor posibilidad de adaptarse a los cambios, pero no están exentos de presentar niveles de estrés con dicha contingencia. De modo, que el afrontamiento no efectivo de los estresores en medio del confinamiento podría acarrear trastornos de conducta, agresividad, bajo rendimiento académico, actitud negativa, mala comunicación, o expresiones de ira u hostilidad ante la percepción de frustración o desesperación en medio del confinamiento (Ribot, Chang, y González, 2020).

En cuanto a la relación entre estrés y resiliencia, los resultados dan cuenta de que en medio de la pandemia, a mayores niveles de resiliencia menos impacto psicosocial de los estresores, o mayor capacidad de adaptación, lo que corrobora la función catalizadora de la resiliencia en tiempos de pandemia. De acuerdo a Cunias y Medina (2021) la resiliencia permite 
neutralizar el impacto psicosocial del estresor lo que disminuye la percepción de los efectos adversos, logrando que los sujetos puedan sobreponerse a la demanda, manteniendo una proyección positiva hacia el futuro. En este sentido, en un grupo de participantes se encontró relación directa entre la resiliencia y las estrategias de afrontamiento de solución de problemas, búsqueda de apoyo social, religión y búsqueda de apoyo profesional, lo que coloca en evidencia la relevancia de los factores externos de la resiliencia en el afrontamiento activo de los adolescentes en la reducción de los efectos psicosociales del Covid.

En lo concerniente a la relación entre resiliencia y estilos de afrontamiento, el estudio encontró que existe una correlación directa estadísticamente significativa y moderada, con los estilos de solución de problemas, búsqueda de apoyo social, religión, búsqueda de apoyo profesional, evitación cognitiva y reevaluación positiva. Lo que concuerda con los hallazgos de Sánchez (2021) el cual, proyecta consideraciones pertinentes para afrontar el covid-19, e indica una mayor eficacia en las estrategias de afrontamiento de expresión y comunicación de emociones e ideas y la búsqueda de apoyo social o profesional; lo que contrasta con los hallazgos de Espada et al. (2020) donde se sostiene que, debido a la multiplicidad de fuentes generadoras de estrés en la pandemia, las estrategias de afrontamiento activas favorecen la generación de soluciones más efectivas al momento de mitigar los efectos del confinamiento, siendo para Fornés, García, Frias \& Rosales (2016) las estrategias de afrontamiento como solución de problemas y autonomía aquellas que reducen más eficazmente la percepción del estrés, así como las consecuencias del mismo.

Sin embargo, el estudio evidencia una correlación baja, directa y estadísticamente significativa entre eventos vitales estresantes y las estrategias de afrontamiento como evitación emocional, reacción agresiva y expresión de la dificultad de afrontamiento, lo que da cuenta de la menor eficacia de estas estrategias al momento de controlar o reducir los estresores psicosociales de la pandemia. Al respecto, se destaca el estudio de Gomes, de Melo, Carvalho, Tavares \& de Melo (2021) enfocado en el análisis de la expresión de emociones en estudiantes adolescentes matriculados en una escuela pública de Brasil durante el periodo de la Pandemia, donde concluyen que los estudiantes al privilegiar estrategias de afrontamiento como la autonomía, reevaluación positiva y solución de problemas al momento de gestionar sus emociones, lograron promover prácticas de autocuidado de su salud mental de manera significativa. 
Frente a la correlación entre eventos vitales estresantes y resiliencia se encontró una relación inversa estadísticamente significativa entre las variables, la cual es baja, y aduce que un segmento poblacional al presentar niveles menores de resiliencia se perciben con mayor intensidad los eventos vitales estresantes; por lo que es conveniente, el fortalecimiento de los factores internos $y$ externos de la resiliencia a fin de promover algunos factores protectores al impacto psicosocial de la pandemia (Rodríguez \& Cardozo, 2021). A su vez, el estudio muestra una correlación directa estadísticamente significativa y moderada entre resiliencia y estrategias de afrontamiento como solución de problemas, búsqueda de apoyo social, religión, búsqueda de apoyo profesional, evitación cognitiva y reevaluación positiva, todas ellas estrategias de afrontamiento activas al momento de enfrentar los eventos psicosociales del Covid, o cualquier demanda psicosocial. En efecto, la dinamización de los recursos internos y externos de la resiliencia en tiempos de pandemia favorece una adaptación positiva ante la adversidad, y beneficia la estabilidad y bienestar psicológico de los adolescentes (Sánchez, Pérez, Alatorre, Aguilera \& Castillo, 2020).

Para finalizar, el estudio de las estrategias de afrontamiento de los adolescentes en torno a eventos vitales estresantes derivados de la emergencia social, sanitaria y ambiental de la pandemia, así como de los procesos de resiliencia, permite identificar la eficacia de las estrategias de afrontamiento activas en la reducción de los estresores de la pandemia; así como la necesidad de fomentar las redes de apoyo social y escenarios para la comunicación y expresión de sentimientos (Pinheiro y Kocourek, 2020), en la promoción de entornos protectores al impacto psicosocial de medidas como el confinamiento, aislamiento selectivo y limitación a la movilidad. De igual manera se evidencia la necesidad entender el impacto psicosocial de la pandemia en perspectiva ecosistémica, lo cual permite retroalimentar las acciones de respuesta del sector educativo de manera holística y no concentrada en el proceso educativo como un hecho aislado. En efecto, es urgente la identificación de las situaciones de adversidad y vulnerabilidad en la que se encuentran inmersos los escolares, de modo que puedan establecerse rutas para el abordaje integral de los efectos psicológicos de la pandemia en la nueva normalidad.

\subsection{Limitaciones de estudio}

Para el desarrollo del estudio se utilizó una muestra intencional, de modo que los participantes se seleccionaron de acuerdo con los criterios de inclusión y 
exclusión establecidos por los investigadores, en este sentido, los resultados caracterizan solo a la muestra seleccionada por lo que se niega la posibilidad de la generalización. Asimismo, dada la contingencia actual, solo fue posible acceder a una muestra de estudiantes entre los 14 a 19 años, por lo que se sugiere que en futuros estudios se aplique los instrumentos a estudiantes de otras etapas etarias para poder analizar las variables de estudio y comparar los hallazgos con los encontrados en la presente investigación.

\section{CONCLUSIONES}

Este artículo muestra un primer acercamiento sobre las variables estrategias de afrontamiento, resiliencia y eventos vitales estresantes en adolescentes escolarizados en tiempos de pandemia, en la ciudad de Pasto, Colombia. Se concluye la existencia de una correlación directa débil entre las variables eventos vitales estresantes y las estrategias de afrontamiento de evitación emocional y reacción agresiva, y una correlación inversa entre eventos vitales estresantes y las estrategias de solución de problemas y búsqueda de apoyo social. En cuanto a las variables eventos vitales estresantes y resiliencia se presenta una relación inversa estadísticamente significativa, con fuerza de correlación baja.

La correlación entre resiliencia y estrategias de afrontamiento es directa, estadísticamente significativa y moderada para las estrategias de solución de problemas, búsqueda de apoyo social, mientras se presenta una correlación inversa moderada entre resiliencia y las estrategias de evitación emocional y expresión de la dificultad de afrontamiento. Estos resultados podrían orientar el diseño de programas psicopedagógicos de abordaje de los eventos vitales estresantes en el retorno a la presencialidad de los establecimientos educativos, y brindan insumos para el estado del arte del impacto psicosocial del Covid-19 en población adolescente en Colombia.

Conflicto de interés: No se presenta ningún conflicto de interés en la investigación que pudiera comprometer los resultados obtenidos.

Agradecimiento: A la Institución Educativa Ciudad de Pasto, Colombia, por las facilidades otorgadas para la ejecución del estudio

Financiamiento: Proyecto de Investigación Afrontamiento Psicológico frente a los Estresores Psicosociales derivados de la Covid-19 en Adolescentes Escolarizados de la Ciudad de Pasto. 


\section{REFERENCIAS}

Alianza para la Protección de la Infancia en Acción Humanitaria. (2020). Nota técnica: Protección de la infancia durante la pandemia de coronavirus. 1-12. Recuperado de https://www.unicef.org/media/66276/file/SPANISH_Technical\%20Note:\%20 Protection\%20of\%20Children\%20during\%20the\%20COVID-19\%20Pandemic.pdf

Álvarez, A. (2020). Clasificación de las investigaciones. Repositorio Institucional-Ulima. Recuperado de https://repositorio.ulima.edu.pe/handle/20.500.12724/10818

Burbano, J. H., \& Guerrero, L. M. (2021). Relación entre factores predisponentes a la deprivación sociocultural y el apoyo social en adolescentes. Revista Virtual Universidad Católica del Norte, (63), 39-62. https://www.doi.org/10.35575/ rvucn.n63a3

Castaño. E. \& del Barco, B., (2010). Estrategias de afrontamiento del estrés y estilos de conducta interpersonal. International Journal of psychology and psychological therapy, 10(2), 245-257. Recuperado de https://www.redalyc.org/ pdf/560/56017095004.pdf

Congreso de Colombia. (6 de septiembre del 2006). Ley 1090 de 2006. DO: 46.383. Recuperado de https://www.funcionpublica.gov.co/eva/gestornormativo/ norma.php?i=66205

Coronado, A. (2016). Academic resilience: a transcultural perspective. Procedia Social and Behavioral Sciences. Recuperado de https://idus.us.es/bitstream/ handle/11441/60611/Comunicaci\%F3n1Preprint.pdf?sequence=7

Cunias, M. \& Medina, I. (2021). Resiliencia en estudiantes adolescentes de una institución educativa de una zona urbano-marginal durante el covid-19. Revista de Investigación y Cultura, 10 (1), 31-39. Recuperado de http://revistas.ucv.edu.pe/ index.php/UCV-HACER/article/view/2703/2166

Delgado, C. \& Santacruz, M. (2011). Caracterización psicométrica del cuestionario de eventos vitales estresantes ceve-ar para adolescentes de Pasto. (Tesis de Pregrado). Universidad de Nariño. Nariño, Colombia. Recuperado de http://sired. udenar.edu.co/3987/1/86017.pdf

Espada, J., Orgilés, M., Piqueras, J. \& Morales, A. (2020). Las buenas prácticas en la atención psicológica infanto-juvenil ante el COVID-19. Clínica y Salud, 31(2), 109-113. https://doi.org/10.5093/clysa2020a14.

Fornes, J., Garcia. G., Frias, D. \& Rosales, G. (2016). Coping, stress, and personality in Spanish nursing students: A longitudinal study. Nurse education today, (36), 318-323. https://doi.org/10.1016/j.nedt.2015.08.011

García, F. \& Acuña, L. (2016). Relación entre experimentar eventos vitales estresantes y su severidad. Interamerican Journal of Psychology, 50(3), 380-391. Recuperado de https://www.redalyc.org/pdf/284/28450492007.pdf

Gomes, A, de Melo, C., Carvalho, J., Tavares, M. \& de Melo, M. (2021). Emoções manifestas por adolescentes escolares na pandemia COVID-19. Research, Society and Development, 10(3), 1-6. Recuperado de https://www.rsdjournal.org/index. $\mathrm{php} / \mathrm{rsd} /$ article/view/13179

Gómez, G. \& Rivas, M. (2017). Resiliencia académica, nuevas perspectivas de interpretación del aprendizaje en contextos de vulnerabilidad social. Calidad en la educación, (47), 215-233. Recuperado de https://scielo.conicyt.cl/pdf/caledu/ n47/0718-4565-caledu-47-00215.pdf

Hernández, R., Fernández, C. \& Baptista, L. (2014). Metodología de la investigación 6 edición. México: McGraw-Hill. Recuperado de http://observatorio.epacartagena. gov.co/wp-content/uploads/2017/08/metodologia-de-la-investigacion-sexta-edicion.compressed.pdf 
Leiva, A., Nazar, G., Martinez-Sanguinetti, M., Petermann-Rocha, F., Ricchezza, J. \& Celis-Morales, C. (2020). Dimensión psicosocial de la pandemia: la otra cara del covid-19. Ciencia y enfermería, 26(10), 1-12. Recuperado de https://scielo. conicyt.cl/pdf/cienf/v26/0717-9553-cienf-26-10.pdf

Londoño, N., Henao, G., Puerta I. Posada, S., Arango, D. Aguirre, A. \& Acevedo, D. (2006). Propiedades psicométricas y validación de la escala de estrategias de coping modificada (EEC-M) en una muestra colombiana. Universitas Psychologica, 5(2), pp.327-349. Recuperado de https://www.redalyc.org/pdf/647/64750210. pdf

Lorenzo, A., Díaz, K. \& Zaldívar, D. (2020). La psicología como ciencia en el afrontamiento a la COVID-19: apuntes generales. AACC, 10(2). 1-8. Recuperado de http://www.revistaccuba.cu/index.php/revacc/article/view/839

Ministerio de salud y Protección social (2020). Salud para jóvenes y adolescentes en época de pandemia. Recuperado de https://www.minsalud.gov.co/Paginas/ Salud-para-jovenes-y-adolescentes-en-epoca-de-pandemia.aspx

Montes, J. (2020). El duelo al mundo de ayer: la vida en la escuela después del COVID-19. Psicología en contextos de COVID-19. 169. Recuperado de https:// ascofapsi.org.co/pdf/Psicologia-contextos-COVID-19_web.pdf

Obando, L., Narváez, J. \& Crespi, M. (2020). Resiliencia, exposición a la violencia y rendimiento académico en un grupo de adolescentes de la ciudad de Pasto, Colombia. [Manuscrito no publicado]. Departamento de Psicología, Universidad de Buenos Aires.

Organización Mundial de la Salud (2020). La OMS caracteriza a COVID-19 como una pandemia. Recuperado de https://www.paho.org/arg/index.php?option=com_ content\&view=article\&id=10436:la-oms-caracteriza-a-covid-19-como-una-pandemia\&Itemid $=226$

Pérez, V., Alcázar, R. \& Reidl, M. (2020). Las estrategias de afrontamiento que utilizan los adolescentes ante situaciones que provocan miedo. Psicogente, 20(38), 240-255. Recuperado de http://revistas.unisimon.edu.co/index.php/psicogente/ article/view/2544

Pinheiro, W. \& Kocourek, S. (2020). Salud mental en tiempos de pandemia: ¿cuál es el impacto de Covid-19? Revista Cuidarte, 11(3). 1-4. https://doi.org/10.15649/ cuidarte.1250

Prime, H., Wade, M., \& Browne, D. T. (2020). Risk and Resilience in Family Well-Being During the COVID-19 Pandemic. American Psychologist, 75(5), 631-643. https:// doi.org/10.1037/amp0000660

Rey, M. (2015). Adaptación escolar, social y familiar, estrés cotidiano y acontecimientos vitales estresantes en la infancia. (Tesis de doctorado). Universidad complutense de Madrid. España, Recuperado de https://eprints.ucm.es/37537/1/T37176.pdf

Reyes, V., Reséndiz, A., Alcázar, R. \& Reidl, L. (2017). Las estrategias de afrontamiento que utilizan los adolescentes ante situaciones que provocan miedo. Psicogente, 20(38), 240-255. http://doi.org/10.17081/psico.20.38.2544

Ribot, V., Chang, N. \& González, A. (2020). Efectos de la COVID-19 en la salud mental de la población. Revista Habanera de Ciencias Médicas, 19(1), 53-64. Recuperado de https://www.redalyc.org/pdf/798/79825836002.pdf

Rodríguez, M. \& Cardozo, I. (2021). Resiliencia en estudiantes adolescentes de una institución educativa de una zona urbano-marginal durante el covid-19. UCV-HACER: Revista de Investigación y Cultura, 10(1), 31-40. Recuperado de https://dialnet.unirioja.es/servlet/articulo?codigo=7946120 
Saavedra, E. \& Villalta, M. (2008). Medición de las características resilientes: un estudio comparativo en personas entre 15 y 65 años. Liberabit, 14(14), 32-40. Recuperado de http://www.scielo.org.pe/pdf/liber/v14n14/a05v14n14

Sánchez, C., Pérez, G., Alatorre, B. Aguilera, P. \& Castillo, B. (2020). Envir on mental Influence on Adolescent Resilience Development in Medellin. Aquichan, 20(2), 20-24. https://doi.org/10.5294/aqui.2020.20.2.4

Sánchez, I. (2021). Impacto psicológico de la COVID-19 en niños y adolescentes. Medisan, 25(1). 123-141. Recuperado de http://scielo.sld.cu/scielo.php?scrip$\mathrm{t}=\mathrm{sci}$ _abstract\&pid=S1029-30192021000100123\&lng=es\&nrm=iso

Sandín, B., García, V. \& Chorot, P. (2020). Impacto psicológico de la pandemia de COVID-19: Efectos negativos y positivos en población española asociados al periodo de confinamiento nacional. RPPC, 25(1), 1-22. Recuperado de http:// revistas.uned.es/index.php/RPPC/article/view/27569

Shigemura, J., Ursano, R., Morganstein, M, \& Benedek, D. (2020). Public responses to the novel 2019 coronavirus (2019-nCoV) in Japan: Mental health consequences and target populations. Psychiatry and Clinical Neurosciences, 74(4), 281-282. https://doi.org/10.1111/pcn.12988

Solis, C. \& Vidal, A. (2006). Estilos y estrategias de afrontamiento en adolescentes. Revista psiquiátrica y salud mental Hermilio Valdizan, 7(1), 33-39. Recuperado de https://www.mdp.edu.ar/cssalud/deptoterapia/archivos/Psicologia_General_ SEMINARIO/ESTILOS_Y_ESTRATEGIAS_DE_AFRONTAMIENTO_en_adolescencia. pdf

Tremolada, M., Bonichini, S. \& Taverna, L. (2016). Coping Strategies and Perceived Support in Adolescents and Young Adults: Predictive Model of Self-Reported Cognitive and Mood Problems. Psychology, 7(14), 1858-1871. http://doi:10.4236/ psych.2016.714171

Uribe, A., Ramos, I., Villamil, I. \& Palacio, J. (2018). La importancia de las estrategias de afrontamiento en el bienestar psicológico en una muestra escolarizada de adolescentes. Psicogente, 21(40), 440-457. https://doi.org/10.17081/psico.21.40.3082.

Villalobos, F., Delgado, M., Santacruz, M. \& Timarán, C. (2011). Caracterización psicométrica del cuestionario de eventos vitales Estresantes CEVE-A-R para adolescentes de Pasto. (Tesis de pregrado). Universidad de Nariño, Nariño, Colombia. Recuperado de http://sired.udenar.edu.co/3987/1/86017.pdf

Viñas, F., Malo, S., González, M. \& Navarro, D. (2015). Assessing mindfulness on a sample of Catalan-speaking Spanish adolescents: Validation of the Catalan version of the child and adolescent mindfulness measure. The Spanish journal of psychology, (18), 1-8. https://doi.org/10.1017/sjp.2015.48

Esta obra está bajo: Creative commons attribution 4.0 international license. El beneficiario de la licencia tiene el derecho de copiar, distribuir, exhibir y representar la obra y hacer obras derivadas siempre y cuando reconozca y cite la obra de la forma especificada por el autor o el licenciante.

\section{(cc) BY}

